\title{
Mediciones frecuentes en el sistema nervioso central mediante tomografía computada e imágenes de resonancia magnética
}

\section{Frequent Measurements in the Central Nervous System by Computed Tomography and Magnetic Resonance Imaging}

\author{
Pablo Sartori ${ }^{10}$ Luis Alvarado ${ }^{2}$ María Chirveches ${ }^{2}$ Macarena Urrutia ${ }^{3}$ Braian Yampolsky ${ }^{1}$ \\ ${ }^{1}$ Diagnóstico Mediter, Sanatorio Julio Méndez, Ciudad Autónoma de \\ Buenos Aires, Argentina \\ Address for correspondence Pablo Sartori, Avenida Avellaneda 551, \\ ${ }^{2}$ TCba. Centro de Diagnóstico, Ciudad Autónoma de Buenos Aires, \\ Ciudad Autónoma de Buenos Aires, Argentina
}

Argentina

${ }^{3}$ Instituto de Investigaciones Neurológicas Dr. Raúl Carrera (FLENI),

Ciudad Autónoma de Buenos Aires, Argentina

Rev Argent Radiol 2020;84:9-16.

\section{Resumen \\ Palabras Clave \\ - sistema nervioso central \\ - tomografía computada \\ - imágenes de resonancia magnética}

En la práctica diaria, durante la realización de informes, suelen presentarse variantes anatómicas, lesiones y diversas patologías del sistema nervioso central (SNC) en las que es necesario realizar algún tipo de mediciones con la finalidad de formular un diagnóstico preciso.

Esas mediciones permiten al médico derivante o tratante programar y realizar procedimientos terapéuticos mínimamente invasivos o que requieran una intervención quirúrgica de mayor relevancia.

Realizamos una descripción de las mediciones más frecuentes en el SNC empleando tomografía computada (TC) e imágenes de resonancia magnética (RM).

On daily practice, during the making of reports, anatomical variants often appear, as well as injuries and different pathologies of the central nervous system (CNS) in which it is necessary to take some kind of measurements, to make an accurate diagnosis. These measurements allow the practicing physician to program and perform minimally invasive therapeutic methods or others that request a surgery of greater relevance. We describe the most frequent measurements in the NCS using Computed Tomography (CT) or Magnetic Resonance Imaging (MRI).

\section{Introducción}

El preciso conocimiento de la anatomía y morfología de las estructuras que conforman el sistema nervioso central (SNC) permite, en la práctica diaria, la realización de informes con una descripción detallada, así como el reconocimiento de variantes anatómicas y diferentes patologías que se presenten. received

February 24, 2019

accepted

November 18, 2019
DOI https://doi.org/ $10.1055 / \mathrm{s}-0040-1701274$. ISSN 1852-9992.
Copyright (c) 2020, Sociedad Argentina de Radiología. Publicado por Thieme Revinter Publicações Ltda., Rio de Janeiro, Brazil. Todos los derechos reservados.
License terms

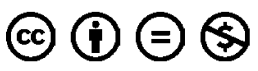


La medición de dichas estructuras en condiciones normales permite categorizar y reconocer medidas anormales cuando se presenten.

El objetivo del presente trabajo es describir e ilustrar las mediciones más frecuentes, aportando información detallada a los colegas derivantes, colaborando con un diagnóstico certero y aportando datos que permitan elegir la terapéutica adecuada.

Las mediciones más utilizadas en los estudios de neuroimágenes son:

\section{Cuantificación del volumen hemático}

Los accidentes cerebrovasculares (ACV) de tipo hemorrágico constituyen una causa frecuente de consultas en urgencias, representando el $10 \%$ de los ACV totales, afectando a 37.000 pacientes al año en los Estados Unidos. ${ }^{1,2}$

La causa principal de los ACV hemorrágicos es la hipertensión arterial. ${ }^{1}$

La presencia de sangrado intracerebral está asociado con un $40 \%$ de mortalidad al mes de producido y un $60 \%$ deriva en discapacidad con necesidad de cuidados permanentes posteriores. $^{1-3}$

Conocer el volumen de sangrado es un factor pronóstico fundamental y determinante en el tratamiento y evolución del mismo. Permite además predecir la morbi-mortalidad del paciente. $^{1}$

Mientras mayor sea el volumen de sangrado, peor será el pronóstico del paciente, especialmente cuando se asocia a deterioro neurológico. ${ }^{1}$

Generalmente, en los informes de neuroradiología suele remarcarse la existencia de sangrado, localización y lateralidad, pudiendo extenderse en la presencia de efecto de masa y edema asociado, pero no suelen especificarse las medidas o el volumen del mismo. La fórmula $A B C / 2$ constituye un valor fiable y certero de cuantificación de volumen de sangrado para realizar en un escaneo de tomografía computada (TC) multiplanar en una emergencia., ${ }^{1,2}$

\section{Método $A B C / 2$}

La medición del volumen de un hematoma intraparenquimatoso se realiza mediante un método denominado $\mathrm{ABC} / 2$ que está basado en el cálculo de volumen de un elipsoide o esfera. ${ }^{2,4-7}$

Fue reportado por primera vez por Kwak. ${ }^{8}$

Para llevar a la práctica este método de medición del volumen de los hematomas intraparenquimatosos mediante una TC de cerebro, se elige el corte con el área de mayor sangrado, midiéndose el máximo diámetro anteroposterior del mismo (parámetro A). El parámetro $\mathrm{B}$ corresponde al diámetro transversal de la lesión en ese mismo corte. ${ }^{3,4,7} \mathrm{El}$ parámetro $\mathrm{C}$ es el número de cortes en los que se aprecia sangrado multiplicado por el espesor de corte ( $\mathbf{F i g . 1}$ ). 1,3,4,7

La fórmula es: A x B x C / 2 y el resultado final se expresa en centímetros cúbicos. ${ }^{1}$

Estudios de cohorte recomiendan el tratamiento quirúrgico en los pacientes con un volumen de sangrado mayor a $25 \mathrm{~cm}^{3}$; en los casos de lesiones de menor volumen la indicación es variable, dependiendo del estado clínico del paciente. ${ }^{4}$
El método $\mathrm{ABC} / 2$ puede ser empleado también para los hematomas extra o subdurales. ${ }^{4}$

Se consideran signos de riesgo de deterioro neurológico a las colecciones intraparenquimatosas superiores a $25 \mathrm{~cm}^{3}$, colecciones subdurales mayores de $1 \mathrm{~cm}^{3}$, aumento de la presión intracraneal, desplazamiento de la línea media superior a $5 \mathrm{~mm}$, dilatación del ventrículo contralateral al hemisferio de la lesión hemorrágica, obliteración de las cisternas media y perimesencefálicas o del III ventrículo. ${ }^{4}$

El método $\mathrm{ABC} / 2$ permite cuantificar rápidamente el volumen de un hematoma sin necesidad de software especializado, pudiendo ser realizado con el paciente en la sala de urgencias. Sin embargo, algunos autores consideran el método poco específico, ya que puede subvalorar el volumen del hematoma, especialmente en aquellos sangrados de bordes irregulares, con contornos polilobulados o que no tienen forma elíptica, pudiendo considerarse otras técnicas de volumetría (planimetría) asistida por computadoras que incorporan un software especial. ${ }^{1,3-7}$

\section{Medición de la línea media}

La presencia de una lesión (intra o extra-axial) con efecto de masa, puede generar desplazamiento de la línea media, provocando herniaciones, compresión de cisternas basales, aumento de la presión intracraneal y llevando al óbito. ${ }^{8,9}$

Este desplazamiento puede mesurarse por TC o resonancia magnética (RM) en cortes axiales, tomando como referencia la posición de las principales estructuras mediales como: septum pellucidum, hoz del cerebro, III ventrículo o glándula pineal. ${ }^{8,9}$

Para medirlo, se traza una línea recta que pase a través de la hoz cerebral desde su inserción rostral hasta la inserción dorsal en la tabla interna. ${ }^{9-11}$

En caso de que una lesión genere efecto de masa y desplace la línea media, se desplazarán las estructuras mediales anteriormente mencionadas. Para mesurar ese desplazamiento, se traza una línea perpendicular a la línea media hasta donde se encuentra el septum pellucidum o la hoz cerebral y se mide la distancia (- Fig. 2). ${ }^{9-11}$

Un desplazamiento de la línea media mayor a $0,5 \mathrm{~cm}$ es un predictor de mal pronóstico para la evolución neurológica de pacientes con traumatismos de cráneo internados en terapia intensiva. $^{12,13}$

\section{Valoración de la herniación o descenso de las amigdalas cerebelosas}

Las amígdalas cerebelosas son estructuras ovoideas localizadas en la superficie inferior del cerebelo, inmediatamente cefálicas al agujero occipital. Deben su nombre a su parecido con las almendras. $^{14}$

Las causas del descenso de las amígdalas son varias, incluyendo: aumento de presión intracraneal, mielomeningocele, malformación de Chiari, hipoplasia de la fosa posterior, escoliosis idiopática, entre otras. ${ }^{14}$

La ectopia de las amígdalas cerebelosas es considerada como el desplazamiento inferior de las mismas cuando es menor o igual a $5 \mathrm{~mm} .^{14,15}$ 

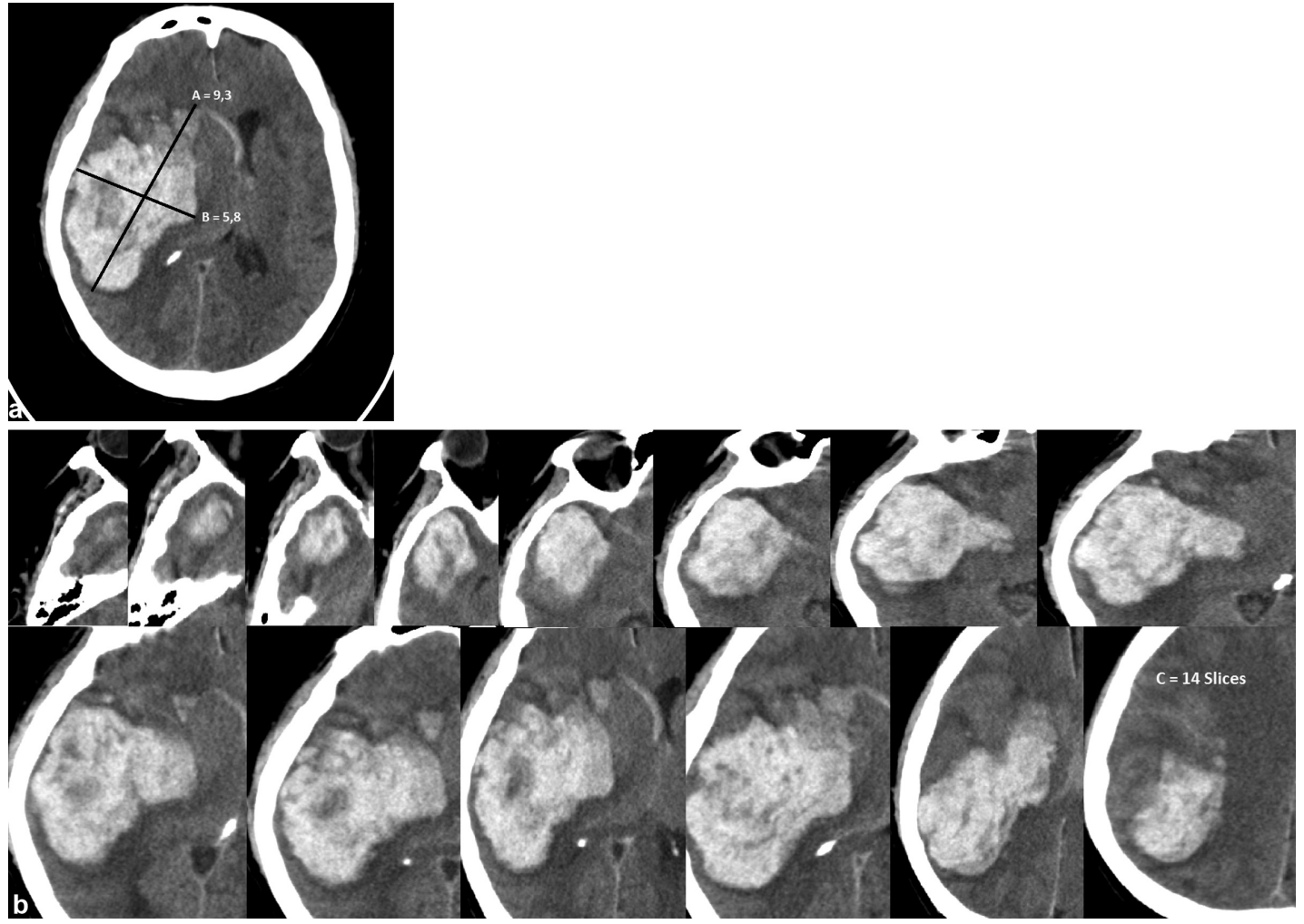

Fig. 1 Medición de hematoma. Método $A B C / 2$. Los cortes axiales son los empleados para esta medición. TC de cerebro en la que se aprecia voluminoso hematoma fronto-temporo-parietal derecho, con efecto de masa desplazando la línea media con volcado ventricular asociado. En (a) se miden: el máximo diámetro antero-posterior $(A=9,3)$ y el diámetro máximo transversal del hematoma $(B=5,8)$. En (b) se muestra el número total de cortes en los que se aprecia el sangrado que correspondería a $C=14$ multiplicado por el espesor de corte $0,5 \mathrm{~cm}$, lo que correspondería a $C=7$. Extrapolando la ecuación quedaría: $A B C / 2: 9,3 \times 5,8 \times 7 / 2=188,7 \mathrm{~cm}^{3}$.

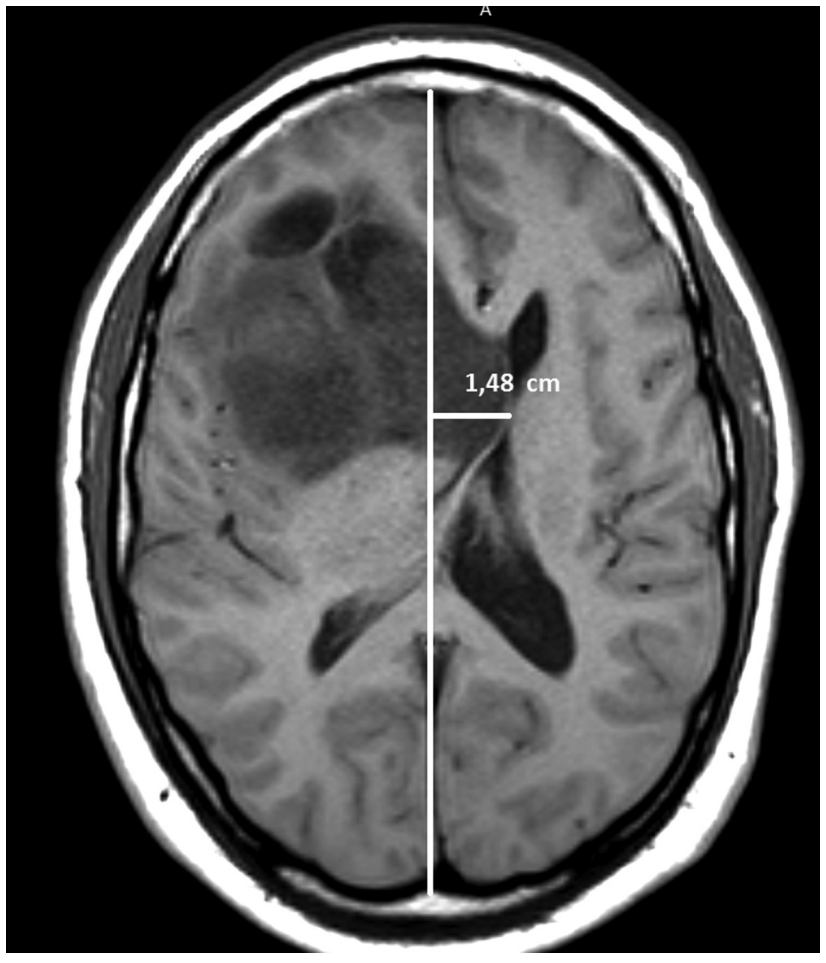

Fig. 2 La medición de la línea media se realiza con cortes axiales de TC o RM. RM de cerebro. Corte axial ponderado en T1, en el que se observa un glioblastoma frontal derecho con efecto de masa, desplazando la línea media hacia la izquierda en aproximadamente $1,48 \mathrm{~cm}$.
Un descenso mayor a $5 \mathrm{~mm}$ es considerado una variante de la malformación de Chiari. ${ }^{14,16}$

La RM es la modalidad de elección, prefiriéndose los cortes sagitales para la medición, ya que en los coronales se dificulta la visualización de los límites del agujero Magno. ${ }^{17}$

La medida debe tomarse trazando una línea recta entre el basión y el opistión del agujero Magno. Luego se traza una línea perpendicular a la primera, hasta el extremo distal de la amígdala herniada (-Fig. 3) ${ }^{18-20}$

Existe disenso entre los estudios de cohorte en relación al valor exacto en milímetros del descenso amigdalar. La - Tabla 1 resume las medidas del descenso amigdalar según el grupo etario. ${ }^{20}$

Existe relación entre el grado de descenso amigdalino y la presencia y grado de sintomatología, ${ }^{17}$ descensos menores a $10 \mathrm{~mm}$ suelen no dar síntomas. ${ }^{19}$

\section{Valoración de la atrofia encefálica}

Tanto la TC como la RM se emplean para la valoración de mediciones estandarizadas que permiten calcular o estimar la evolución de algunas enfermedades generalmente relacionadas con desórdenes del movimiento o con la demencia. ${ }^{21,22}$

Los índices bicaudado, de Evans y bifrontal se obtienen en cortes axiales de TC y RM con orientación orbito-meatal, utilizándose para mesurar el envejecimiento fisiológico y cuantificar el grado de atrofia cerebral (- Tablas 2-4). ${ }^{22-24}$ 
Mediciones frecuentes en el sistema nervioso central mediante tomografía computada e imágenes de resonancia
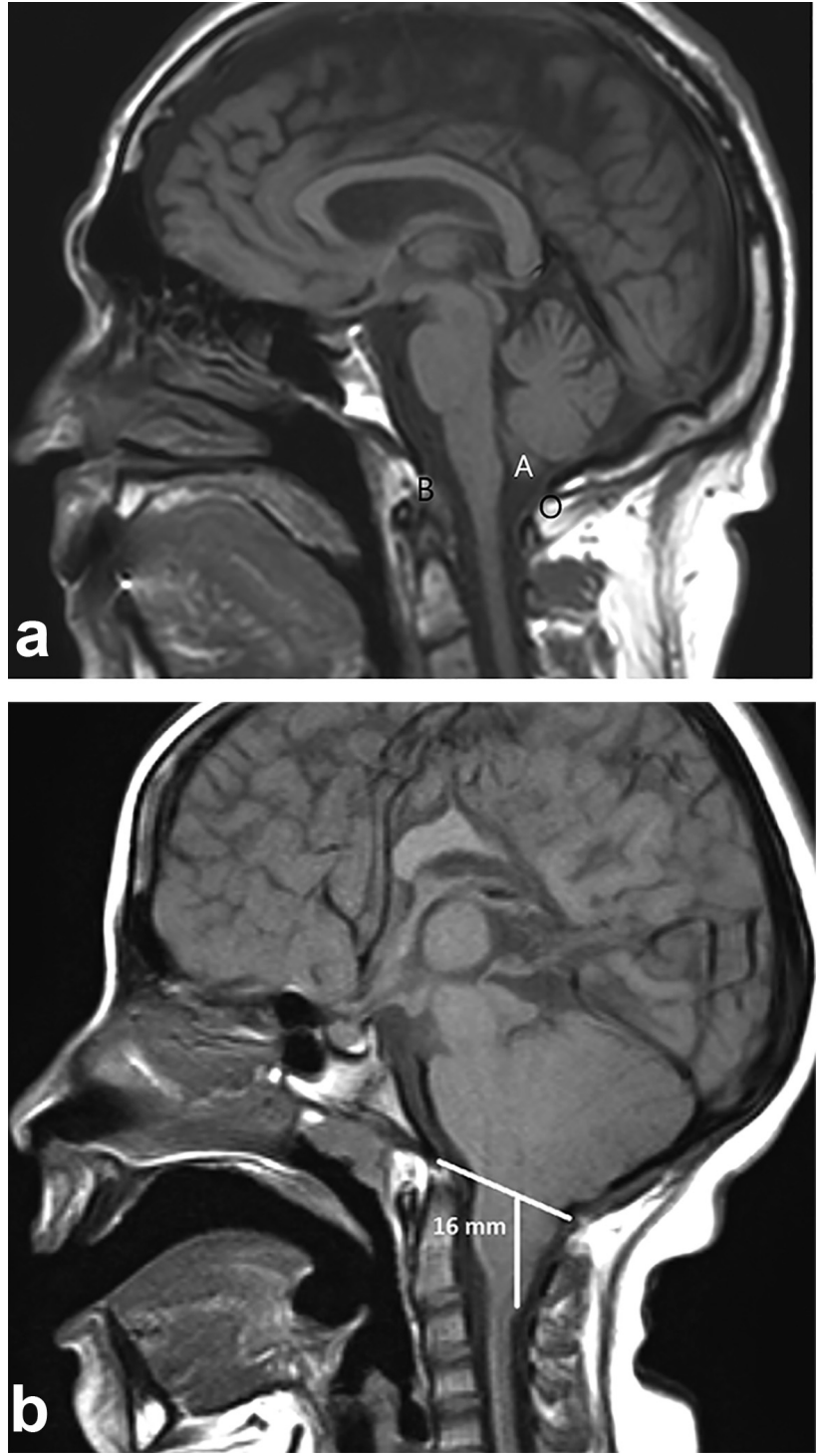

Fig. 3 (a) Los cortes sagitales, de preferencia en RM, son los usados para valorar el descenso amigdalar. RM en secuencia ponderada en T1 de la unión cráneo-cervical. Se marcan el Basión (B), Opistion (O) y las amígdalas cerebelosas (a) de localización normal. (b) RM de cerebro, corte sagital pornderado en $\mathrm{T} 1$ en paciente con malformación de Arnold-Chiari II. Se aprecia descenso de las amígdalas cerebelosas a través del foramen Magno en $16 \mathrm{~mm}$. Se acompañó de siringomielia (no mostrada).

Tabla 1 Descenso normal de las amígdalas cerebelosas, según $\operatorname{edad}^{20}$

\begin{tabular}{|l|l|}
\hline Décadas & $\begin{array}{l}\text { Descenso en } \mathrm{mm} \\
\text { considerado normal }\end{array}$ \\
\hline Primera & 6 \\
\hline Segunda y tercera & 5 \\
\hline Cuarta a octava & 4 \\
\hline Novena & 3 \\
\hline
\end{tabular}

A) Índice bicaudado: se obtiene dividiendo la distancia mínima de los ventrículos laterales a nivel de las cabezas de los núcleos caudados con el diámetro del cráneo a ese mismo nivel (-Fig. 4).
Tabla 2 Valores normales de los índices, distribuidos por sexo (incluyendo desvío standard) $^{22}$

\begin{tabular}{|l|l|l|l|}
\hline Índice & Sexo masculino & Sexo femenino & Valor $\boldsymbol{p}^{*}$ \\
\hline Bifrontal & $0,326+/-0,033$ & $0,319+/-0,031$ & $>0,05$ \\
\hline Bicaudado & $0,132+/-0,040$ & $0,119+/-0,032$ & $<0,05$ \\
\hline Evans & $0,270+/-0,026$ & $0,263+/-0,026$ & $>0,05$ \\
\hline
\end{tabular}

*Los coeficientes de correlación de Pearson obtenidos entre los índices y la edad fueron para el índice bifrontal: $r=0,64$; para el índice bicaudado: $r=0,69$ y para el índice de Evans: $r=0,60$.

Tabla 3 Valores normales de los índices distribuidos por sexo en personas menores o igual a 50 años (incluyendo desvío standard) 22

\begin{tabular}{|l|l|l|l|l|}
\hline Índice & $\begin{array}{l}\text { Sexo } \\
\text { masculino }\end{array}$ & $\begin{array}{l}\text { Sexo } \\
\text { femenino }\end{array}$ & $\begin{array}{l}\text { Promedio } \\
50 \text { años }\end{array}$ & $\begin{array}{l}\text { Valor } \\
\boldsymbol{p}^{*}\end{array}$ \\
\hline Bifrontal & $\begin{array}{l}0,308+l \\
-0,030\end{array}$ & $\begin{array}{l}0,310+l \\
-0,033\end{array}$ & $\begin{array}{l}0,309+/ \\
-0,031\end{array}$ & $>0,05$ \\
\hline Bicaudado & $\begin{array}{l}0,109+/ \\
-0,020\end{array}$ & $\begin{array}{l}0,108+l \\
-0,022\end{array}$ & $\begin{array}{l}0,108+l \\
-0,021\end{array}$ & $>0,05$ \\
\hline Evans & $\begin{array}{l}0,259+/ \\
-0,023\end{array}$ & $\begin{array}{l}0,257+l \\
-0,026\end{array}$ & $\begin{array}{l}0,258+l \\
-0,024\end{array}$ & $>0,05$ \\
\hline
\end{tabular}

*Los coeficientes de correlación de Pearson obtenidos entre los índices y la edad fueron para el índice bifrontal: $r=0,64$; para el índice bicaudado: $r=0,69$ y para el índice de Evans: $r=0,60$.

Tabla 4 Valores normales de los índices distribuidos por sexo en personas mayores de 50 años (incluyendo desvío standard) ${ }^{22}$

\begin{tabular}{|l|l|l|l|}
\hline Índices & Sexo masculino & Sexo femenino & Valor $\boldsymbol{p}^{*}$ \\
\hline Bifrontal & $0,343+/-0,028$ & $0,327+/-0,028$ & $>0,05$ \\
\hline Bicaudado & $0,153+/-0,043$ & $0,129+/-0,036$ & $<0,05$ \\
\hline Evans & $0,281+/-0,024$ & $0,269+/-0,024$ & $>0,05$ \\
\hline
\end{tabular}

*Los coeficientes de correlación de Pearson obtenidos entre los índices y la edad fueron para el índice bifrontal: $r=0,64$; para el índice bicaudado: $r=0,69$ y para el índice de Evans: $r=0,60$.

B) Índice de Evans: se calcula dividiendo el diámetro máximo de las astas frontales de los ventrículos laterales con el diámetro máximo intracraneal en el mismo corte a la altura de los forámenes de Monro (- Fig. 5). ${ }^{22-24}$

Un valor $>$ a 0,30 indica ventriculomegalia y puede considerarse patológico, pero no puede discriminarse la etiología por atrofia $u$ otra entidad. ${ }^{22-24}$

C) Índice bifrontal: se calcula dividiendo el diámetro máximo de las astas frontales de los ventrículos laterales y el diámetro intracraneal a ese mismo nivel (- Fig. 6). ${ }^{22-24}$

\section{Medición del ángulo del pedúnculo cerebral}

Es una medida utilizada para cuantificar la enfermedad neurodegenerativa conocida como síndrome de SteeleRichardson-Olszewski (SSRO). Esa patología se caracteriza por la atrofia selectiva del tronco encefálico, especialmente a nivel de los pedúnculos cerebrales, generando aumento de la 


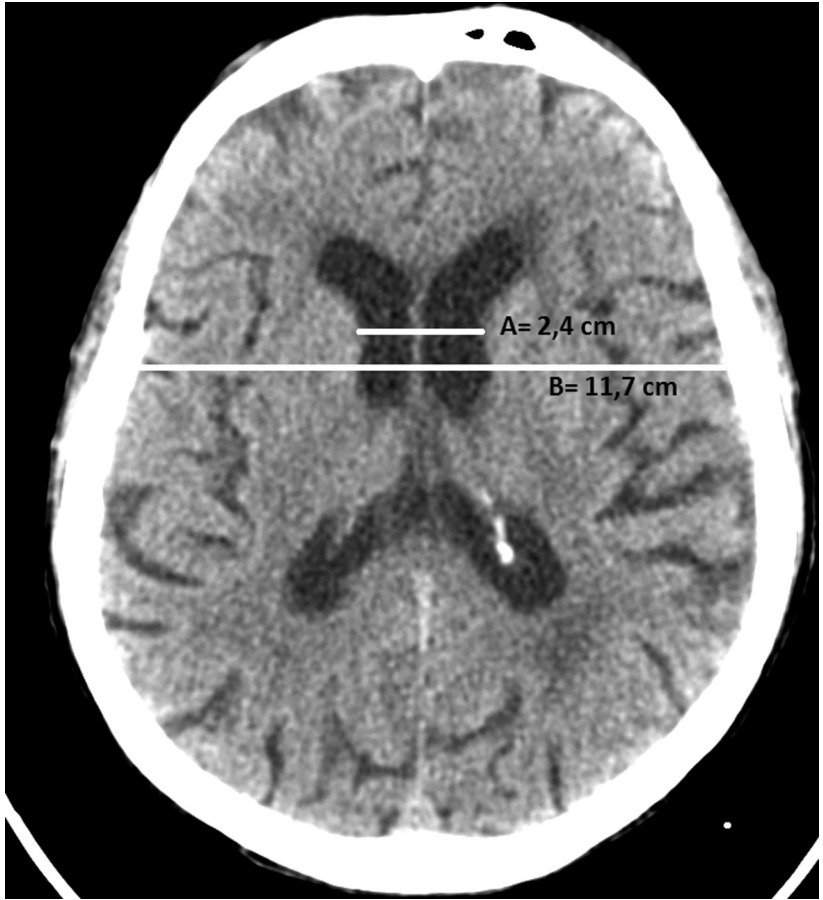

Fig. 4 La medición del índice bicaudado debe realizarse con cortes axiales de TC o RM. TC corte axial de cerebro. Paciente masculino de 78 años con deterioro cognitivo. Se traza una línea recta que mide la distancia entre los bordes mediales de las cabezas de ambos núcleos caudados $(A=2,4 \mathrm{~cm})$. Luego se traza otra línea midiendo el diámetro máximo craneal a ese nivel $(B=11,7 \mathrm{~cm})$. La fórmula sería 2,4\% 11,7= 0,20 indicando atrofia moderada.

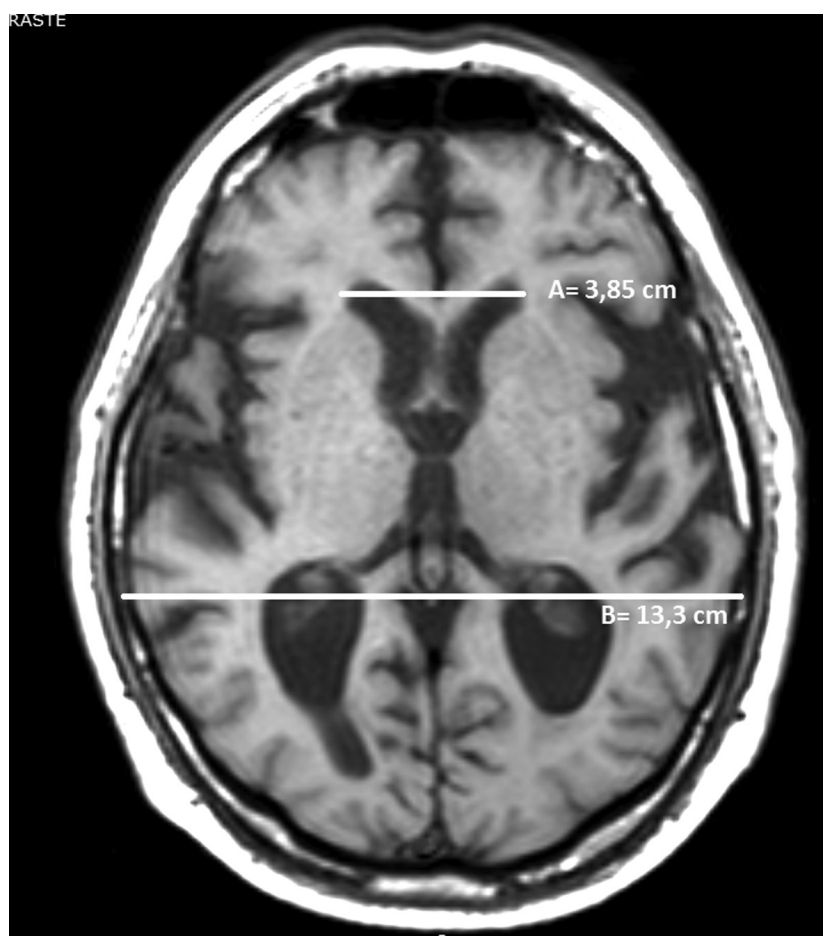

Fig. 5 El índice de Evans se obtiene en cortes axiales tanto en TC como en RM. RM de cerebro. Corte axial en secuencia ponderada en T1. Debe medirse la distancia entre las prolongaciones frontales de los ventrículos laterales $(A=3,85 \mathrm{~cm})$ dividido por el diámetro máximo del cráneo en el mismo corte a la altura de los agujeros de Monro ( $B=13,3 \mathrm{~cm}$ ). Ejemplificando 3,85\% $13,3=0,28$ para una paciente de 63 años con alteración incipiente del desarrollo intelectual.

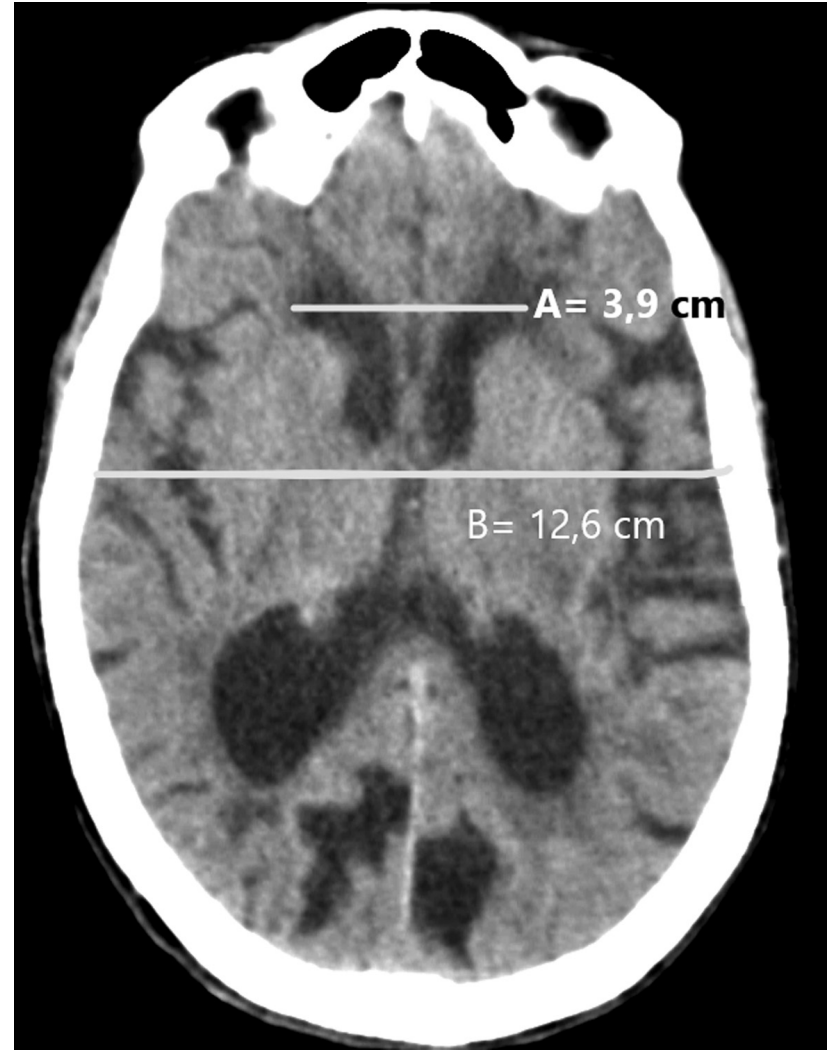

Fig. 6 El índice bifrontal (tal como el de Evans), debe obtenerse en cortes axiales de TC o RM, como se muestra en esta RM ponderada en $\mathrm{T} 1$, en la que se mide la distancia entre las prolongaciones frontales de los ventrículos laterales, dividido por el diámetro máximo intracraneal a ese nivel. En nuestro ejemplo sería: 3,9\% 12,6=0,30, indicando un valor normal para un paciente de 63 años.

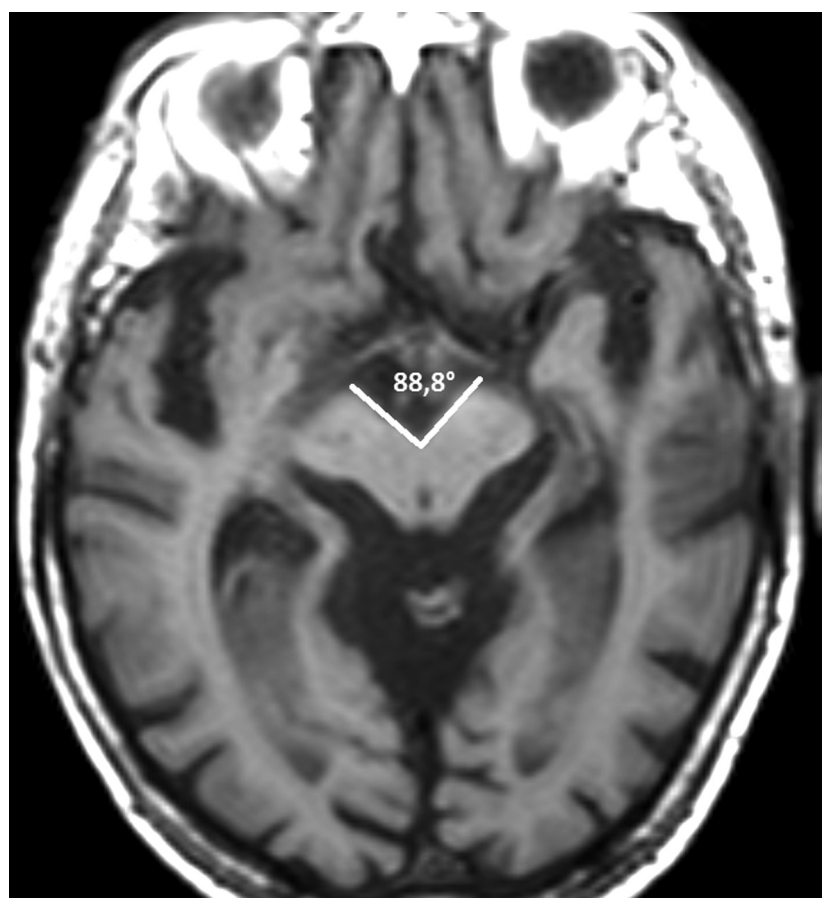

Fig. 7 Los cortes axiales de TC o RM sin oblicuar, son los empleados para la medición del ángulo del pedúnculo cerebral. RM de cerebro, secuencia ponderada en T1: paciente con diagnóstico de SSRO hace tres años. El ángulo del pedúnculo cerebral es de $88,8^{\circ}$, acorde con valores para su enfermedad de base. 
Mediciones frecuentes en el sistema nervioso central mediante tomografía computada e imágenes de resonancia

apertura en la cisterna interpeduncular valorada en secuencias axiales de TC o RM. ${ }^{24}$

En un corte axial de una RM de cerebro, a la altura de los tubérculos mamilares, se trazan dos líneas paralelas a los bordes mediales de los pedúnculos cerebrales hasta que contacten en su vértice, midiendo el ángulo que forman (-Fig. 7). ${ }^{2}$

Un valor de ángulo de pedúnculo cerebral mayor o igual a $62^{\circ}$ permite corroborar el diagnóstico de SSRO, diferenciándolo de otras patologías neurodegenerativas con un menor grado de apertura, como lo es el síndrome de Parkinson $\left(53-54^{\circ}\right.$ ) o la Atrofia multisistémica $\left(55-56^{\circ}\right) .^{24}$
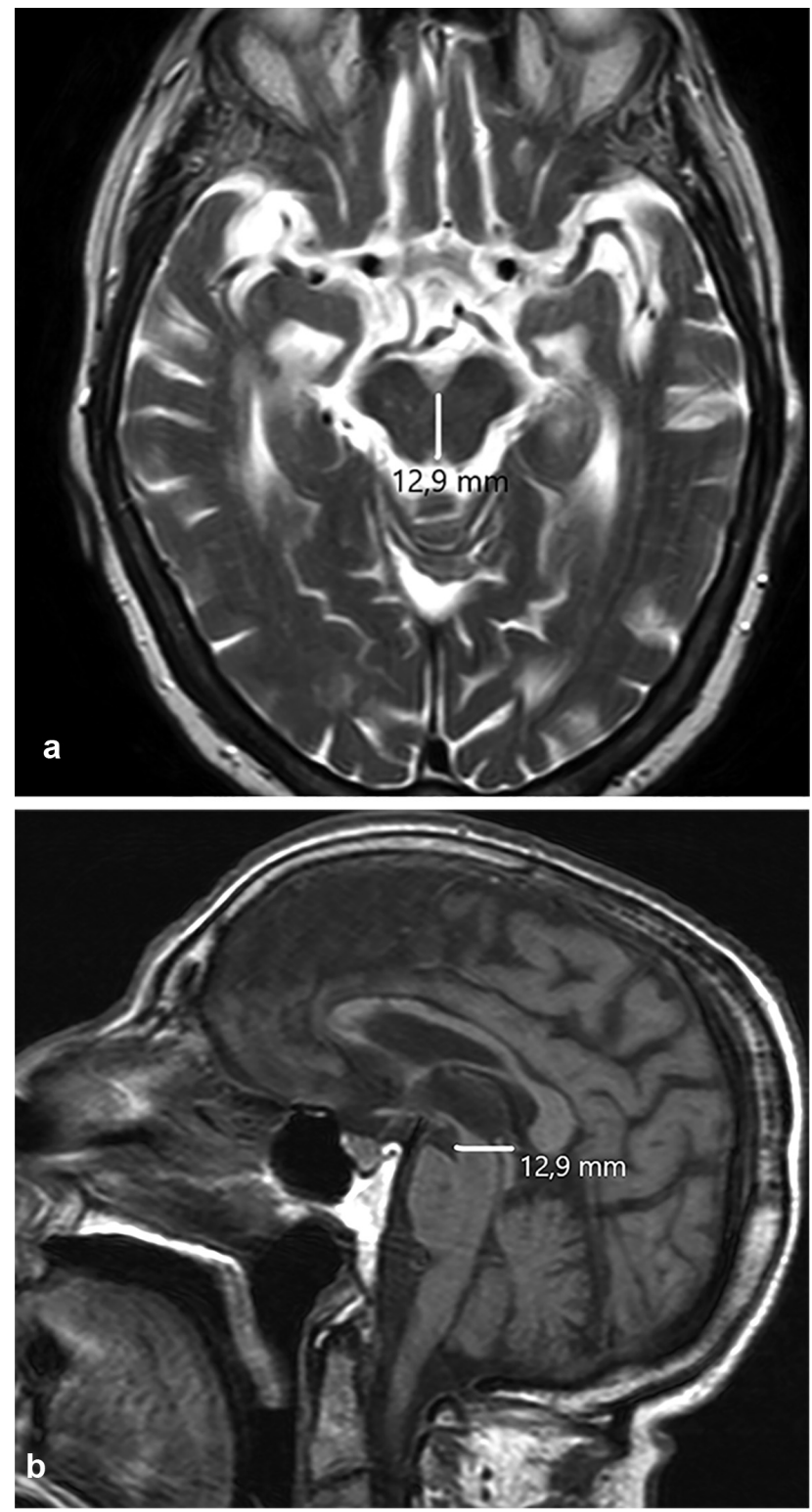

Fig. 8 El diámetro antero-posterior del mesencéfalo se efectúa en RM a nivel de los colículos superiores en el corte axial ponderado en T2 (a) y sagital ponderado en T1 (b), en un paciente con sospecha clínica de SSRO. Obsérvese la configuración característica en "cabeza de Mickey Mouse” del mesencéfalo (a). El diámetro antero-posterior de los pedúnculos es de $12,9 \mathrm{~mm}$, medida que indica marcada atrofia mesencefálica y confirma el diagnóstico de SSRO.

\section{Medición del diámetro antero-posterior del mesencéfalo}

La reducción del diámetro anteroposterior del mesencéfalo a la altura de los tubérculos cuadrigéminos superiores (colículos), es característica del SSRO, originando una configuración típica en "cabeza de Mickey Mouse" (-Fig. 8). ${ }^{25,26}$

Esa medida se obtiene en cortes axiales ponderados en T2 o sagitales ponderados en T1 de RM, trazando una línea desde el borde ventral del mesencéfalo, a la altura de los colículos superiores, hasta el borde dorsal. ${ }^{25}$

El diámetro antero-posterior aproximado del mesencéfalo en pacientes con SSRO suele ser menor a $14 \mathrm{~mm}$; valores superiores permiten descartar SSRO. ${ }^{25}$

\section{Valoración del ancho del asta temporal en la enfermedad de Alzheimer}

Existen diversos parámetros en imágenes para valorar el grado de atrofia o involución del parénquima encefálico en las diversas enfermedades neurodegenerativas, como por ejemplo en la enfermedad de Alzheimer.

Esas medidas son indicadores que demuestran la progresión de enfermedad.

Existe involución del parénquima encefálico a nivel de la circunvolución hipocampal, amígdala y corteza entorrinal. ${ }^{21}$

Para valorarla, se pueden emplear tanto TC como RM midiendo el ancho del asta temporal de los ventrículos laterales para comparar en estudios posteriores el avance de la atrofia encefálica. ${ }^{21}$

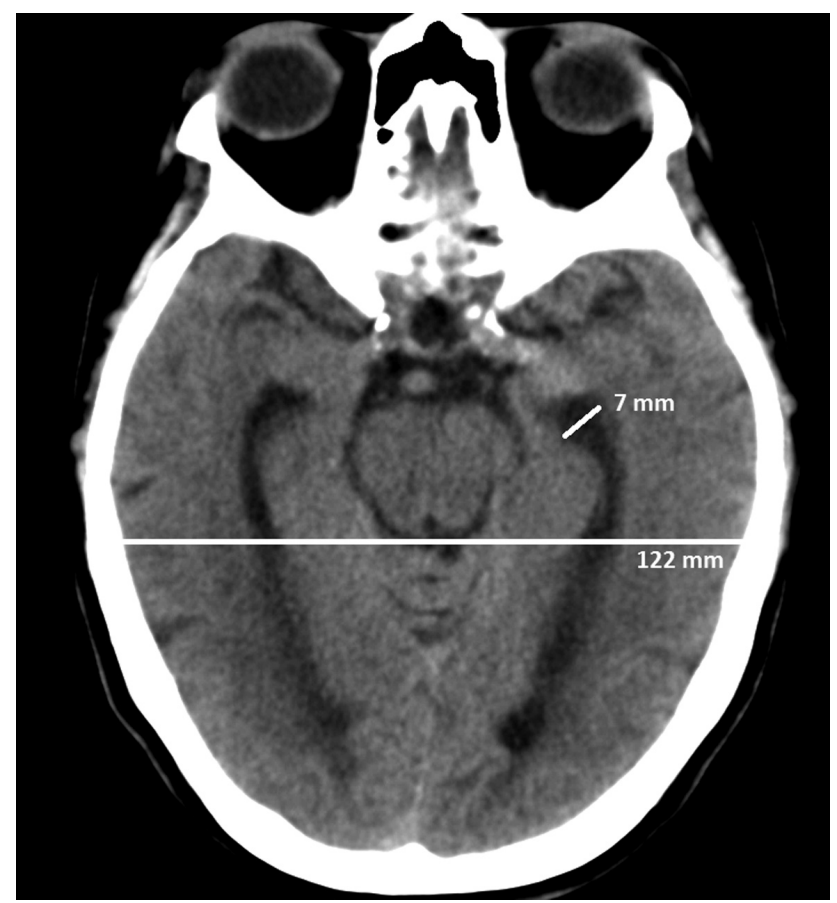

Fig. 9 Paciente con sospecha clínica de Alzheimer. Para la medición del asta temporal pueden emplearse cortes axiales de TC o RM. Se realiza TC para valorar el ancho del asta temporal del los ventrículos laterales, que miden $7 \mathrm{~mm}$. El diámetro máximo intracraneal es de $122 \mathrm{~mm}$. El cociente entre ambos es de 0,05 , siendo compatible con el diagnóstico presuntivo. 


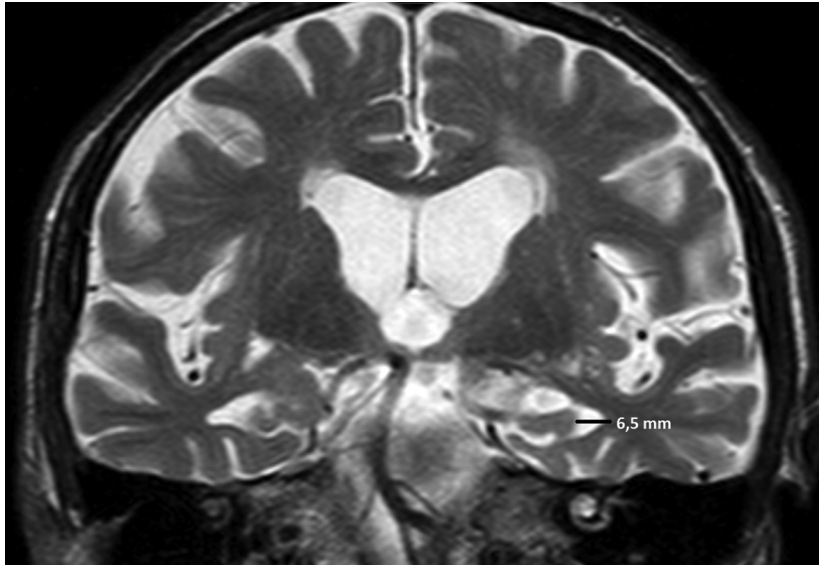

Fig. 10 Los cortes coronales en RM permiten lograr una precisa medición de la prolongación temporal del ventrículo lateral. Corte coronal ponderado en T2 a la altura de la prolongación temporal del ventrículo lateral. La distancia del ancho del asta es de $6,5 \mathrm{~mm}$, en relación a la enfermedad de Alzheimer leve.

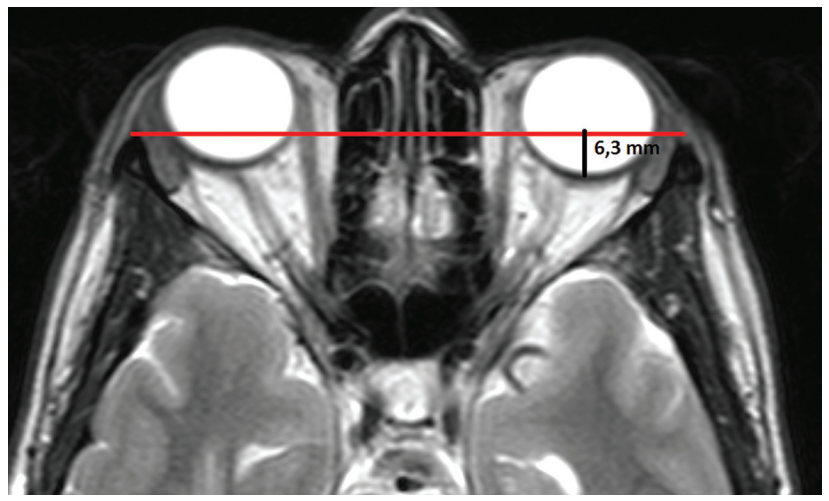

Fig. 11 Tanto la TC como la RM en cortes axiales a nivel de las órbitas deben ser consideradas para lograr una correcta medición. RM de órbitas, corte axial potenciada en T2. Para valorar la proptosis debe trazarse una línea entre las apófisis cigomáticas (línea roja), luego se realiza una línea perpendicular a la anterior hasta la esclera posterior (línea negra). Valores mayores a $9,9 \mathrm{~mm}$ son compatibles con proptosis. En nuestro ejemplo, la distancia es inferior por lo que es considerado como normal.

Se mide en un corte axial de TC el diámetro máximo del asta temporal, luego se toma en el mismo corte el máximo diámetro biparietal para realizar el cociente entre ambas medidas (-Fig. 9). ${ }^{21}$

En personas normales, el valor es de 0,025 . En pacientes con Alzheimer es de 0,038 y de 0,044 en pacientes con Alzheimer asociado a extensas lesiones en la sustancia blanca. ${ }^{21}$

En RM se emplea un corte coronal (siempre en un mismo nivel) para medir el ancho del asta temporal (-Fig. 10). ${ }^{21}$ En un estudio de cohorte en pacientes mayores de 60 años estimaron valores del asta temporal de $6,6 \mathrm{~mm}$ en la enfermedad de Alzheimer leve y 7,2 mm para la avanzada. ${ }^{21}$

\section{Proptosis}

La proptosis es el desplazamiento o protrusión anterior del globo ocular. El término exoftalmos es empleado como sinónimo de proptosis, aunque suele relacionarse con las oftalmopatías endócrinas. ${ }^{27}$

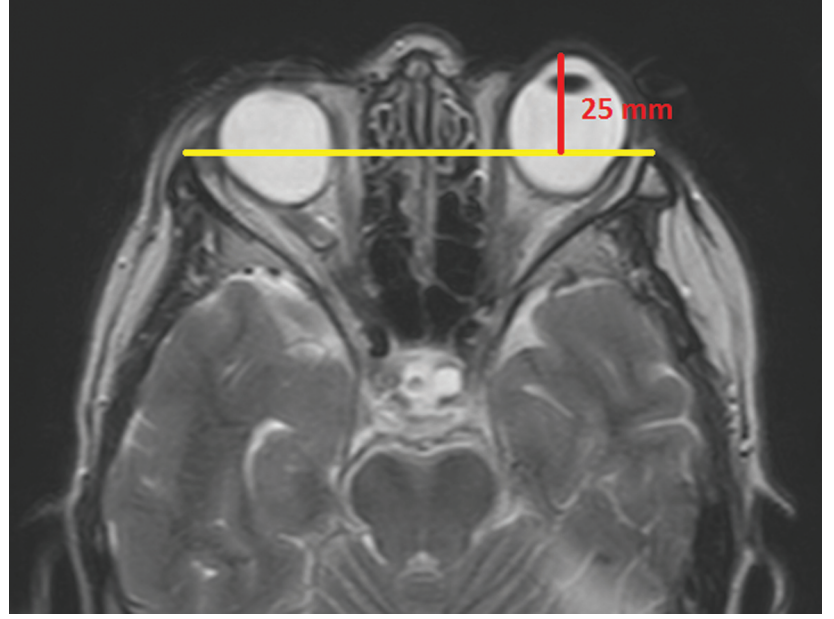

Fig. 12 Otra forma de valorar si existe proptosis mediante cortes axiales de TC o RM, es el que se muestra en esta RM en secuencia ponderada en T2, en la que se realiza la línea intercigomática (línea roja), luego se traza otra línea perpendicular a la misma hasta el borde anterior del globo ocular, considerándose normal hasta $21-23 \mathrm{~mm}$. Nuestro ejemplo lo supera $(25 \mathrm{~mm})$, por lo que existe proptosis.

La propotsis puede cuantificarse empleando cortes axiales de TC o RM, donde se aprecie al globo ocular en sus diámetros máximos (incluso en forma prenatal). ${ }^{27-30}$

Para ello, se traza una línea recta entre los dos procesos cigomáticos (línea intercigomática). Luego se realiza otra línea (perpendicular a la intercigomática) hacia la esclera posterior: el valor promedio normal es $9,9+/-1,7 \mathrm{~mm}$ (-Fig. 11). ${ }^{27-30}$

La distancia entre la línea intercigomática y el borde anterior del globo ocular debe ser menor a 21-23 mm (- Fig. 12). ${ }^{27-30}$

\section{Responsabilidades Éticas}

Protección de personas y animales. Los autores declaran que para esta investigación no se han realizado experimentos en seres humanos ni en animales. Confidencialidad de los datos. Los autores declaran que han seguido los protocolos de su centro de trabajo sobre la publicación de datos de pacientes.

Derecho a la privacidad y consentimiento informado. Los autores declaran que en este artículo no aparecen datos de pacientes.

\section{Conflicto de Intereses}

Los autores declaran no tener ningún conflicto de interés, excepto el Dr. Pablo Sartori, que declara como posible conflicto de interés ser parte del Capítulo de Neuroradiología de la Sociedad Argentina de Radiología.

\section{Bilbiografía}

1 Maeda AK, Aguiar LR, Martins C, Bichinho GL, Gariba MA. Hematoma volumes of spontaneous intracerebral hemorrhage: the ellipse $(A B C / 2)$ method yielded volumes smaller than those measured using the planimetric method. Arq Neuropsiquiatr. 2013;71(08):540-544

2 Zimmerman RD, Maldjian JA, Brun NC, Horvath B, Skolnick BE. Radiologic estimation of hematoma volume in intracerebral 
hemorrhage trial by CT scan. AJNR Am J Neuroradiol. 2006;27 (03):666-670

3 Webb AJ, Ullman NL, Morgan TC, Muschelli J, Kornbluth J, Awad IA, et al; MISTIE and CLEAR Investigators. Accuracy of the ABC/2 Score for Intracerebral Hemorrhage: Systematic Review and Analysis of MISTIE, CLEAR-IVH, and CLEAR III. Stroke. 2015;46 (09):2470-2476

4 Arikan F, Sahuquillo J, Ibáñez J, Vilalta J, Poca MA, Riveiro M, et al. Variabilidad en las indicaciones quirúrgicas de las lesiones intradurales postraumáticas. Neurocirugia (Astur). 2005;16(02): 108-116

5 Wang CW, Juan CJ, Liu YJ, Hsu HH, Liu HS, Chen CY, et al. Volumedependent overestimation of spontaneous intracerebral hematoma volume by the $\mathrm{ABC} / 2$ formula. Acta Radiol. 2009;50 (03):306-311

6 Kleinman JT, Hillis AE, Jordan LC. ABC/2: estimating intracerebral haemorrhage volume and total brain volume, and predicting outcome in children. Dev Med Child Neurol. 2011;53(03): 281-284

7 Hu TT, Yan L, Yan PF, Wang X, Yue GF. Assessment of the ABC/2 Method of Epidural Hematoma Volume Measurement as Compared to Computer-Assisted Planimetric Analysis. Biol Res Nurs. 2016;18(01):5-11

8 Gebel JM, Sila CA, Sloan MA, Granger CB, Weisenberger JP, Green $\mathrm{CL}$, et al. Comparison of the $\mathrm{ABC} / 2$ estimation technique to computer-assisted volumetric analysis of intraparenchymal and subdural hematomas complicating the GUSTO-1 trial. Stroke. 1998;29(09):1799-1801

9 Liao CC, Chen YF, Xiao F. Brain Midline Shift Measurement and Its Automation: A Review of Techniques and Algorithms. Int J Biomed Imaging. 2018;2018:4303161

10 Escario JA, Martínez Quiñones JV, Gallego AM, Arregui Calvo R, Suárez Mier MP. Hernias encefálicas. Clasificación, neuropatología y problemas medicolegales. Rev Esp Med Legal. 2015;41(03):91-102

11 Hacking C. Midline shift. Disponible en: https://radiopaedia.org/ articles/midline-shift. Accedido el 12 de Julio de 2019

12 Motuel J, Biette I, Srairi M, Mrozek S, Kurrek MM, Chaynes P, et al. Assessment of brain midline shift using sonography in neurosurgical ICU patients. Crit Care. 2014;18(06):676-683

13 Chiewvit P, Tritakarn SO, Nanta-aree S, Suthipongchai S. Degree of midline shift from CT scan predicted outcome in patients with head injuries. J Med Assoc Thai. 2010;93(01):99-107

14 Mavridis I. Gross and neurosurgical anatomy of the cerebellar tonsil. OA Anatomy. 2014;2(01):8-13

15 Luijkx T, Gaillard F. Chiari I malformation. Disponible en: https:// radiopaedia.org/articles/chiari-i-malformation. Accedido el 2 de Junio de 2019
16 Jiménez-Caballero PE, Fermín-Marrero JA. Cefalea orgasmica secundaria a malformacion de Chiari tipo 1 resuelta tras cirugia descompresiva. Rev Neurol. 2014;59(01):47-48

17 Barkovich AJ, Wippold FJ, Sherman JL, Citrin CM. Significance of cerebellar tonsillar position on MR. AJNR Am J Neuroradiol. 1986; 7(05):795-799

18 Furuya K, Sano K, Segawa H, Ide K, Yoneyama H. Symptomatic tonsillar ectopia. J Neurol Neurosurg Psychiatry. 1998;64(02):221-226

19 Aparici F, Menor F, Matí Bonmatí L, Miguel A, Pamies J, Sancho A. Herniación amigdalina en la malformación de Chiari en la edad pediátrica: observación y evolución en el tiempo. Radiologia. 2001;43(05):243-248

20 Mikulis DJ, Díaz O, Egglin TK, Sánchez R. Variance of the position of the cerebellar tonsils with age: preliminary report. Radiology. 1992;183(03):725-728

21 Arana Fernández de Moya E. Demencias e Imagen: lo básico. Radiologia. 2010;52(01):4-17

22 Vargas López DA, Rodríguez Alvarez JC. Indice Bicaudado, Indice Bifrontal e Indice de Evans en Tomografías Cerebrales Normales. Revi Peru de Radiol. 2008;12(26):27-29

23 Factora R, Luciano M. Normal pressure hydrocephalus: diagnosis and new approaches to treatment. Clin Geriatr Med. 2006;22(03): 645-657

24 Fatterpekar GM, Dietrich A, Pantano P, Saba L, Knopp EA, Piattella MC, et al. Cerebral Peduncle Angle: An Objective Criterion for Assessing Progressive Supranuclear Palsy Richardson Syndrome. AJR Am J Roentgenol. 2015;205(02):386-391

25 Warmuth-Metz M, Naumann M, Csoti I, Solymosi L. Measurement of the midbrain diameter on routine magnetic resonance imaging: a simple and accurate method of differentiating between Parkinson disease and progressive supranuclear palsy. Arch Neurol. 2001;58(07):1076-1079

26 Patro SN, Glikstein R, Hanagandi P, Chakraborty S. Role of neuroimaging in multidisciplinary approach towards NonAlzheimer's dementia. Insights Imaging. 2015;6(05):531-544

27 Sobrino Guijarro B, Alcazar A, Ordóñez González A, Montoya Bordón J, Alonso Torres A, Lingam R. Revisión radiológica de las causas de proptosis ocular. Disponible en: https://posterng.netkey.at/esr/ viewing/index.php?module=viewing_poster\&task=viewsection $\&$ ti=363029. Accedido el 15 de Junio de 2019

28 Nugent RA, Belkin RI, Neigel JM, Rootman J, Robertson WD, Spinelli J, et al. Graves orbitopathy: correlation of CT and clinical findings. Radiology. 1990;177(03):675-682

29 Bell D, Hameed A. Proptosis. Disponible en: https://radiopaedia. org/articles/proptosis-1 ?lang=us. Accedido el 12 de junio de 2019

30 Freitas Soares Machado K, Mattos García M. Thyroid ophthalmopathy revisited. Radiol Bras. 2009;42(04):261-266 